\title{
SPR Imaging Label-Free Control of Biomineral Nucleation!?
}

\author{
Stancu Izabela-Cristina \\ Politehnica University of Bucharest, Faculty of Applied Chemistry and Materials Science \\ Romania
}

\section{Introduction}

Bone regeneration due to trauma and pathology, bone degeneration in aging population as well as osteporosis ellicit a societal need for new performant regenerative products. Although a wide range of biomaterials have been researched and applied for hard tissue repair and regeneration, there is still a high competition and challenge in improving their performances for in vivo applications.

To create high-quality orthopaedic implants, several approaches have been proposed; among them the self-biomineralizing scaffolds are intensively researched. Despite intensive studies and an impressive number of investigated structures, no optimum formulation has been yet set-up. This is why high-performing methods of controlling the biomineralization induction are still needed and researched. In this context, a deeper and broader understanding of the mineralization phenomenon induced on/inside different materials would be extremely useful.

The present chapter is aimed as the first attempt of investigation of biomaterials-induced biomineralization through the label-free Surface Plasmon Resonance Imaging (SPRi).

\subsection{Biomineralization inductive materials}

One of the most exciting and researched class of biomaterials for bone repair and regeneration is represented by polymer materials supporting or even better, enhancing mineral phase formation (Kamei et al., 1997; Shin et al., 2003; Stancu et al., 2004). The main advantage of these materials over classical bone fillers consists in their in situ transformation in polymer-hydroxyapatite composites, similar to natural bone. The advantages of the soformed composites are evident when compared to synthetic composites prepared prior to implantation; the most important is that the hydroxyapatite is generated inside the bone tissue enhancing the further osteointegration of the implant. The mineralization of either natural or synthetic matrix is an extremely complex phenomenon. To date, there is no consensus over the nucleation processes responsible for materials' calcification (calcification is synonym with biomineralization when referring to bone mineral phase formation). All the hypotheses and the mechanisms researched have lead to a very important common conclusion: the sine-qua-non condition for biomineralization to occur is the presence of calcium and phosphate ions in the surrounding liquid environment. Calcium cations are provided by cells and physiological fluids while phosphate anions are generated through phosphoesters and phosphoproteins hydrolysis as well as from the body fluids (Whyte et

Source: Intelligent and Biosensors, Book edited by: Vernon S. Somerset, ISBN 978-953-7619-58-9, pp. 386, January 2010, INTECH, Croatia, downloaded from SCIYO.COM 
al., 1995). Mineralization to occur needs nucleation to be induced by a perturbation like the ions' capturing by special molecules/species.

Based on the observation that natural phosphate-containing proteins are actively involved in naturally occurring mineralization and due to their calcium affinity, phosphatecontaining polymers have been studied with respect to hydroxyapatite formation in physiological fluids (Stancu et al., 2004; Swart et al., 1976). The literature also presents the carboxylic functionalities from bone proteins (e.g. bone sialoprotein) and from some polymers as responsible for hydroxyapatite formation (Hunter et al., 2001; Filmon et al., 2002; Ganns et al., 1999).

To resume, negatively charged groups seem to elicit hydroxyapatite nucleation and growth through calcium capturing from body fluids (Vijayasekaran et al., 2000). Another important aspect deciding on the mineralization occurrence concerns the importance of the distribution and of the density of the negative groups inside/outside the support (Stancu et al., 2004).

Recently, starting from the idea that a defined chemical functionality could decide on the nucleation and on the mineral crystals' growth, dendrimers have been investigated as leading to mineral embedding the support materials, with predefined shape and properties (Donners et al., 2003). This hypothesis has opened a wide field of research and the present chapter subscribes this direction. Dendrimers are globular tridimensional macromolecules presenting a high number of branched structural units (dendrons) diverging from a central core. The branches end with superficial end groups which form the exterior functional shell of the dendrimer. This reactive shell may contain a diversity of functional groups: amines, carboxylic acids, alcohols, aldehydes, thiols, etc. The polyamidoamine dendrimers (PAMAM) have nano- dimensions and their immobilization on surfaces would lead to structural features within this dimensional range.

The present work represents an attempt of assessment of the biomineralization capacity of PAMAM dendrimers amino- and carboxylic-terminated immobilized on solid supports.

\subsection{Importance of nano-dimension in bone regeneration}

For a bone graft to be successful, it seems that a biomaterial with superficial nano-features and presenting a superior surface reactivity available for further growth factors and other biospecies to be immobilized could represent real advances. Nanomaterials domain covers a wide range of materials of different natures with dimensions under $100 \mathrm{~nm}$ or presenting structural features (particles, fibers) under this dimensional threshold. Considering the tissue regeneration behavior, nanomaterials have been studied with respect to the proteins and cells interactions. The success of these materials is based on the initially interaction with the proteins controlling the cellular functions. Several recent works report superior bone cells performances associated with nanoroughness and nanophase materials of various nature. Professor Dr. Webster T.J. could be considered as a real promoter of the positive effects of nanomaterials in tissue engineering and reconstruction, the research of his group in the last 7 years proving this affirmation. In this context, it has been constantly reported that these materials increase the recognition of the surfaces by the osteoblasts that are naturally accustomed with the nanostructured components of natural bone (HA crystals, fibrillar collagen etc.) (Price et al., 2003; Ward \& Webster, 2006). To remember, bone is structured on three dimensional levels: 1) macroscopic (cortical, trabecullar), 2) microscopic (Haversian systems) and 3) nanometric (mineral crystals, noncollagenous proteins, collagen fibrils). 


\subsection{SPRi}

SPR is a well known high-sensitive techniques specially used for the assessment of both ultrathin layers of biomolecules as well as for the analysis of biomolecular specific interactions. The principle of SPR is based on the detection of molecules' presence on a modified metallic surface through monitoring the change in the local reflectivity, without using molecular labels. In addition to the SPR spectrometer, the SPRi tool has an excellent efficiency and broader application since it allows for simultaneous and comparative investigation of the whole surface of the sensor in real time. The technique is still underexplored and its potential is far of being totally used. In this aim, the approach of mineralization monitoring through SPRi is perfectly justified and welcome. The technique provides high sensitivity, time-driven analysis of mineral phase formation, accuracy in mimicking the physiological conditions with respect to fluid environment providing and, nevertheless, simple operation.

The present work will try to go deeper in the description of biomineralization induced by PAMAM-coated substrates, as explored by SPRi.

\section{Experiment design}

\subsection{Materials}

Substrate. The biosensor substrate is based on commercially available SPR gold slides (sSens, Netherlands). These sensors $(10 \times 10 \mathrm{~mm})$ are made out of borosilicate glass with a refractive index of 1.51 and $0.3 \mathrm{~mm}$ thick. They are compatible both with common commercial SPR instruments as well as with modular SPR set-up. The gold applied onto the sensor's surface is evaporated following an optimized procedure with respect to gold layer thickness and cristallinity; a thin titanium layer was applied in order to ensure a good adherence of the gold on the glass. Substrates' rinsing with piranha solution (prepared using $75 \%$ concentrated $\mathrm{H}_{2} \mathrm{SO}_{4}$ and $25 \%$ of $\mathrm{H}_{2} \mathrm{O}_{2} 30 \%$ ) should be performed for approximately 1 minute, followed by distilled water cleaning and drying under a nitrogen stream; this treatment should immediately precede the use. (Recommended time for rinsing with piranha is up to 5 minutes, in order to remove the contaminants and to avoid the increase of the superficial roughness (Ulman, 1991).

Thiols. Thiols are needed to chemically immobilize the dendrimer molecules onto the gold surface. Thiols with different chain lengths and presenting amino- or carboxyl- terminal functional groups may be utilized: Cysteamine, 3-Mercaptopropionic acid, 16Mercaptohexadecanoic acid, 11-Mercaptoundecanoic amine etc. Thiols should be used without further purification and as freshly prepared ethanolic solutions $(1 \mathrm{mM})$.

Activation mixture. The activation mixture was described elsewhere (Stancu et al., 2007). Briefly, the carboxylic groups can be activated with $20 \mathrm{mM}$ solution of $\mathrm{N}, \mathrm{N}-(3-$ dimethylaminopropyl)-N'-ethyl-carbodiimide hydrochloride (EDC) and $5 \mathrm{mM}$ solution of N-hydroxysuccinimide (NHS) in phosphate buffer pH7.4.

Sensing phase. PAMAM dendrimers with amino- and carboxylic acid- external reactive shells are used as sensing phase. It should be remembered here that amino-terminal PAMAM is full generation and the acid-PAMAM is half generation according to their synthesis. The synthesis of these macromolecules starts from the ethylediamine (EDA) core, followed by Michael addition of methyl acrylate (MA) and amidation reactions of the resulting ester with large excess of EDA. Repetition of this sequence gives full generation 
$\mathrm{g}=0,1,2,3, \ldots$ (amino terminal groups), while the interruption of this sequence after the Michael addition leads to half generations $g=0.5,1.5,2.5, \ldots$ (carboxyl-ended dendrimers) (Majoros et al., 2004). In this study two generations of PAMAM dendrimers both with amino- as well as with carboxyl- ending functionalities are used as sensing phase. Generations 1.5 and 3.5 are used for the dendrimers with - $\mathrm{COOH}$ functional end-groups (further referred as PAMAM-g1.5 and PAMAM-g3.5). Generations 2 and 4 are selected for the $-\mathrm{NH}_{2}$ ending dendrimers (further referred as PAMAM-g2 and PAMAM-g4). All dendrimers are supplied from Sigma-Aldrich and used as received, as solutions in methanol. Generations 1.5 and 2 present 16 ending groups while generations 3.5 and 4 present 64 terminal groups.

Mother liquor inducing the mineralization. Two abiotic fluids of physiological $\mathrm{pH}$ ( $\mathrm{pH} 7.4)$ are to be used as mother liquors from which the mineral is formed. Both are based on the natural mineral constituents and composition of human plasma (as shown in table 1) and are referred as Synthetic Body Fluid (SBF) and SBF 1.5x according to their composition: SBF has very similar composition as the human plasma while SBF $1.5 \mathrm{x}$ is 1.5 folds more concentrated; the latter is used in order to elicit an accelerated response only for the materials/surfaces proved to induce mineralization when exposed to SBF. Preparing SBF is based on the procedure set-up by Prof. Kokubo (Kokubo et al., 1990) and later used by our group (Stancu et al., 2004).

\begin{tabular}{|l|c|c|c|}
\hline & \multicolumn{3}{|c|}{ Ionic concentration, $\mathrm{mmol} / \mathrm{dm}^{3}$} \\
\hline \multicolumn{1}{|c|}{ Ions } & SBF & SBF 1.5x & Human plasma \\
\hline $\mathrm{Na}+$ & 142.0 & 213.0 & 142.0 \\
\hline $\mathrm{K}+$ & 5.0 & 7.5 & 5.0 \\
\hline $\mathrm{Mg} 2+$ & 1.5 & 2.25 & 1.5 \\
\hline $\mathrm{Ca} 2+$ & 2.5 & 3.75 & 2.5 \\
\hline $\mathrm{Cl}-$ & 147.8 & 221.7 & 103.0 \\
\hline $\mathrm{HCO} 3-$ & 4.2 & 6.3 & 27.0 \\
\hline HPO42- & 1.0 & 1.5 & 1.0 \\
\hline SO42- & 0.5 & 0.75 & 0.5 \\
\hline
\end{tabular}

Table 1. Ionic concentration of the mother liquors used in the study (Kokubo et al., 1990; Stancu et al., 2004); the composition of human plasma (Tsuru et al., 2001; Jaakkola et al., 2004) is indicated as control.

The $\mathrm{pH}$ of the prepared solutions is adjusted to 7.40 , at $36.5^{\circ} \mathrm{C}$, using Tris (Tris(hydroxyamino)methane - $\left.\left(\mathrm{CH}_{2} \mathrm{OH}\right)_{3} \mathrm{CNH}_{2}\right)$ ) and $\mathrm{HCl}$. Perfectly clean reservoirs are used to prepare SBF and SBF1.5x; they should be carefully cleaned with $\mathrm{HCl}$ solutions followed by rinsing with demineralized water prior to use. Table 2 indicates the amount of salts needed to prepare $1 \mathrm{~L} \mathrm{SBF}$; the salts should be dissolved successively in the Tris solution previously prepared in demineralized water. Dissolving is carried out under continuous stirring, at $36.5^{\circ} \mathrm{C}$. The $\mathrm{pH}^{\prime} \mathrm{s}$ adjustement represent the last step of the procedure. The stability of the so-prepared solution ahould be checked prior its utilization. The presence of spontaneously formed precipitate corresponds to contaminated solution inappropriate to be used. Only freshly prepared solutions are recommended to be used. 


\begin{tabular}{|c|c|}
\hline Reagent & Amount needed \\
\hline $\mathrm{NaCl}$ & $7.996 \mathrm{~g}$ \\
\hline $\mathrm{NaHCO}_{3}$ & $0.350 \mathrm{~g}$ \\
\hline $\mathrm{KCl}$ & $0.224 \mathrm{~g}$ \\
\hline $\mathrm{K}_{2} \mathrm{HPO}_{4} \cdot 3 \mathrm{H}_{2} \mathrm{O}$ & $0.228 \mathrm{~g}$ \\
\hline $\mathrm{MgCl}_{2} \cdot 6 \mathrm{H}_{2} \mathrm{O}$ & $0.305 \mathrm{~g}$ \\
\hline $1 \mathrm{kmol} / \mathrm{m}^{3} \mathrm{HCl}$ & $40 \mathrm{~cm}^{3}$ \\
\hline $\mathrm{CaCl}_{2}$ & $0.278 \mathrm{~g}$ \\
\hline $\mathrm{Na}_{2} \mathrm{SO}_{4}$ & $0.071 \mathrm{~g}$ \\
\hline$\left(\mathrm{CH}_{2} \mathrm{OH}\right)_{3} \mathrm{CNH}_{2}$ & $6.057 \mathrm{~g}$ \\
\hline $1 \mathrm{kmol} / \mathrm{m}^{3} \mathrm{HCl}$ & $\begin{array}{c}\text { To adjust the } \\
\text { pH }\end{array}$ \\
\hline
\end{tabular}

Table 2. Receipe for 1L SBF.

\subsection{Methods}

\subsubsection{PAMAM immobilization on the sensor's surface}

PAMAM dendrimers with amino and carboxyl terminal functionalities bearing 16 and respectively 64 reactive end groups are immobilized on gold. The immobilization procedure depends on the ending functionality of the dendrimers. The exterior reactive shell of the selected dendrimers is chemically inactive with respect to gold. Thus, in order to create stable PAMAM coating on gold, self-assembled monolayers (SAMs) of amino- and carboxylended thiols should be first created on gold.

Deposition of Thiol SAMs. This is realized through the direct exposure of neat gold surfaces to $1 \mathrm{mM}$ freshly prepared thiol ethanolic solution (as schematically shown in Fig. 1); the spontaneous adsorption and self-organization of thiols is achieved after 24 hours, at room temperature. Further details on thiols SAMs preparation and characterisation on gold are presented elsewhere (Stancu et al., 2007). Thiol SAMs with acidic or amino terminal functions are realized.

Dendrimers immobilization. Following carboxyl-ended thiol SAMs formation, the immobilization of amino- PAMAM occurs through bioconjugation, after the intermediate activation of carboxylic groups with EDC/NHS mixture, for 30 minutes at room temperature and in the dark (schematically shown in Fig.1). One terminal primary amine from amino-PAMAM reacts with an activated carboxyl from the thiol SAMs creating a chemical linkage. Thus, the sensor surface is chemically coated with amino-PAMAM leading to homogeneous dendrimer layers of different morphology/topography and aminodensity, according to the dendrimers generation. Rinsing with demineralised water is performed to remove unreacted species.

Carboxyl-ended dendrimers are first partially activated using EDC/NHS mixture, for 30 minutes at room temperature and in the dark; then, the amino-ending thiol SAMs are exposed to the activated $\mathrm{COOH}$-bearing macromolecules to create COOH-PAMAM coatings on the sensor's surface (Fig. 2). Rinsing with demineralised water is performed to remove unreacted species.

The morphology and reactivity of the new formed surfaces depend on PAMAM's generation as well as shown in Fig.3.

The so created PAMAM surface-loaded sensors should be further characterised with respect to the surface nanoroughness, dendrimers density and distribution in order to provide the 
quantitative characteristics of the sensing phase, further useful to quantify the mineral nucleation occurrence/evolution. Atomic Force Microscopy and a wide range of chemicophysical methods for dendrimers' quantification are available with this respect but they are not the object of this work.
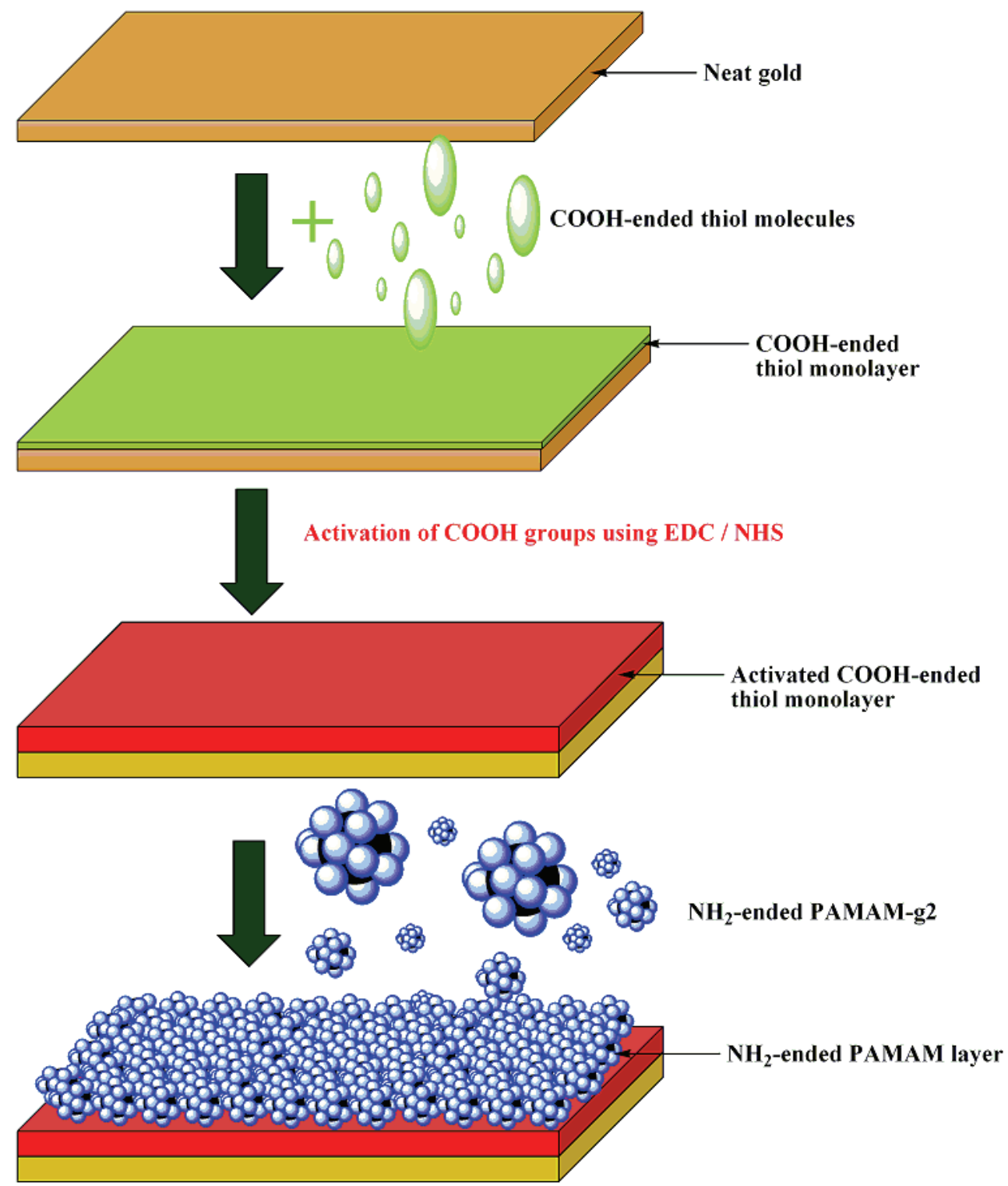

Fig. 1. Design of the $\mathrm{NH}_{2}$-ended PAMAM sensors. Schematic view for PAMAM-g2 


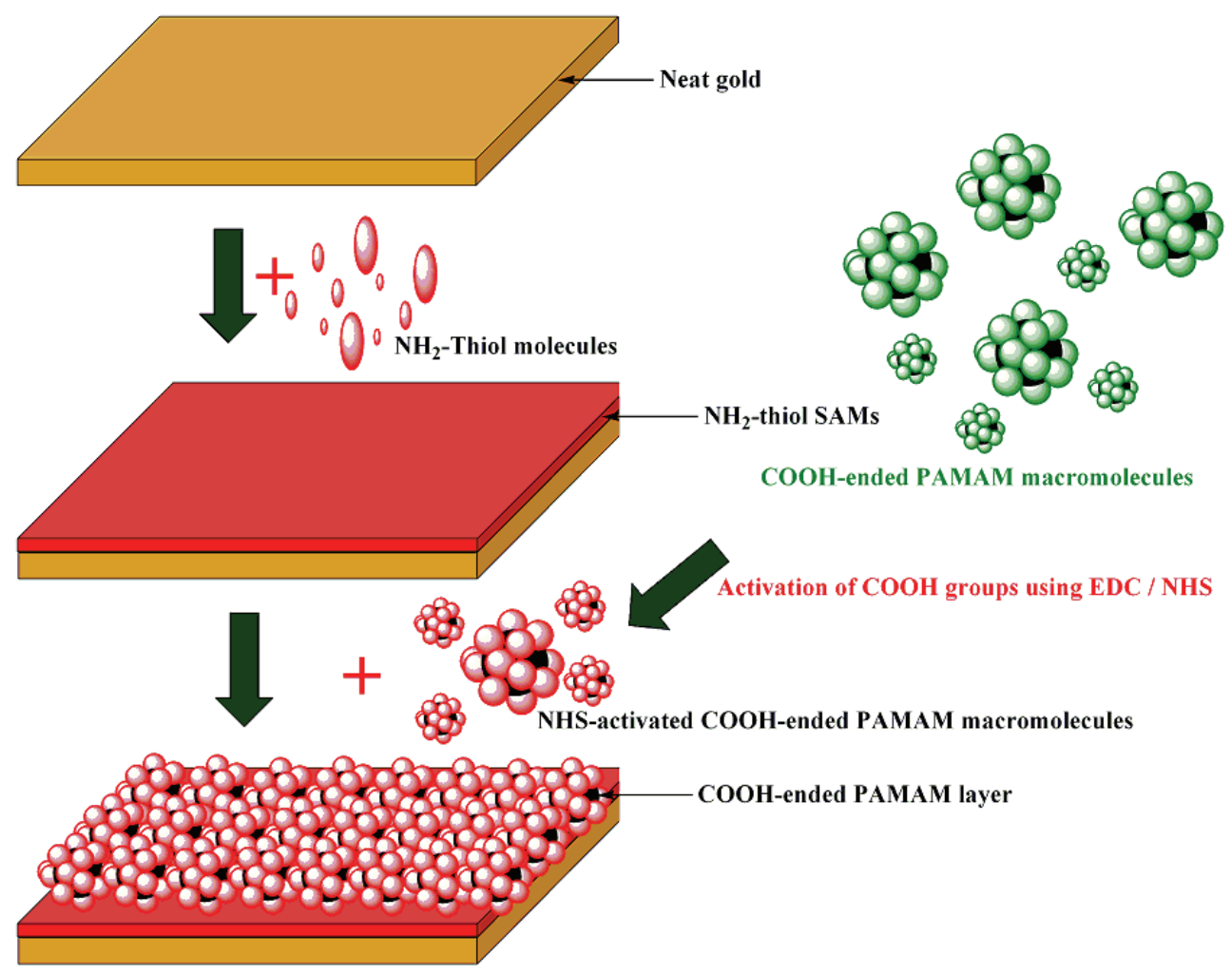

Fig. 2. Design of the COOH-ended PAMAM sensors. Schematic view for PAMAM-g1.5

\subsubsection{SPRi principle and functioning}

The SPRi principle is based on the label-free detection of the whole surface of the array, in a very sensitive manner, regardless the chemical nature of the immobilized molecules. Among commercially available devices one should mention the two GenOptics systems (InteractorTM and SPRiLAB TM) and the GWC SPRImager ${ }^{\circledR I I}$ Array instrument. The researchers in the field of SPR imaging are usually building up their own modular instruments. The modular set-up used in this study was previously described in another work (Stancu et al., 2007); the set-up is presented in Fig. 4. Briefly, monochromatic light (wavelength 632,8 $\mathrm{nm}$ ) is provided by a He-Ne laser LGK 7653-8 (LASOS GmbH, Ebersberg, Germany) (1 in Fig.4). The light beam is p-polyrised through the polaryser ( 2 in Fig. 3 ) and then expanded 10 times using the collimator ( 3 in Fig. 4).

Two tilting mirrors (4 in Fig.4) are guiding the light to the prism, under the convenient incident angle allowing for the SPR occurrence. The system contains a prism (5 in Fig.4) made of BK7 glass ( $n=1.51), 90^{\circ}$. The sensing system (7 in Fig.4) is fixed on the prism surface through a drop of oil (6 in Fig.4) with the same refractive index as the glass of the prism and of the sensor.

The reflected light is guided by another mirror (4 in Fig.4) on the CCD camera (Retiga 1300 Qimaging, Burnaby, British Columbia, Canada) (8 in Fig.4). The collected data are displayed 
in real time and they may be stored for further analysis. For operation in fluid as needed in this work, a flow cell (9 in Fig.4) is used. The circulating fluid is pumped through an inlet and evacuated through an outlet; the flow is $2 \mathrm{ml} /$ minute, as in bone tissue, at $36.5^{\circ} \mathrm{C}$. Operation under continuous flow or under stop-flow mode is possible.

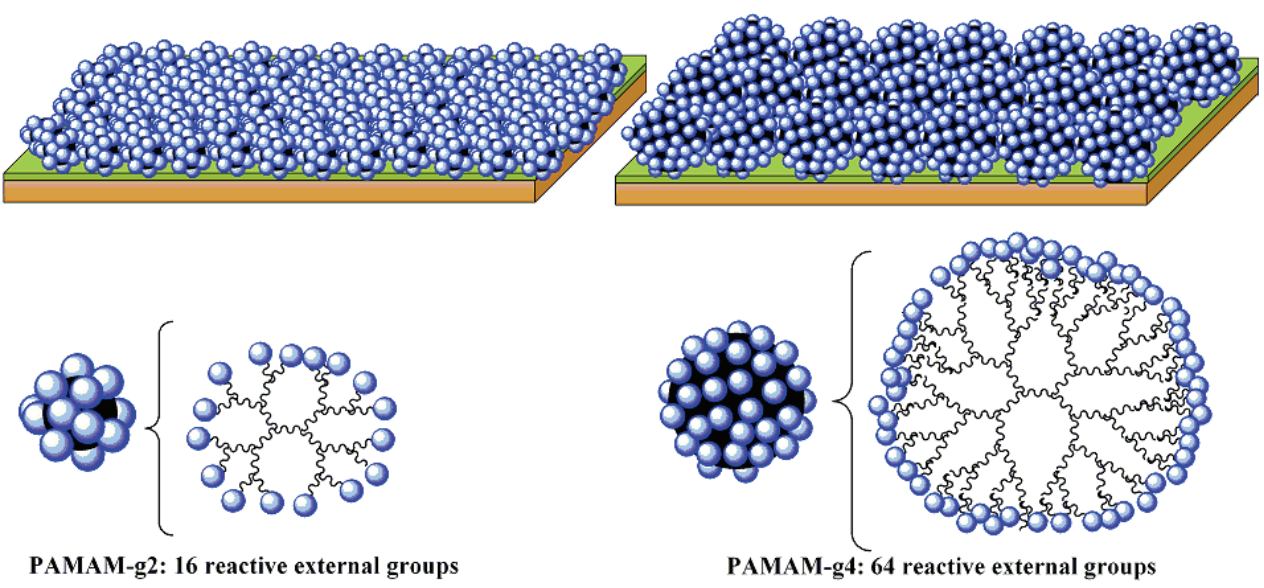

Fig. 3. Schematic view of the surfaces of the sensors coated with amino-terminal PAMAM; the morphology/topography of the sensor's surface depends on the generation of the dendrimer

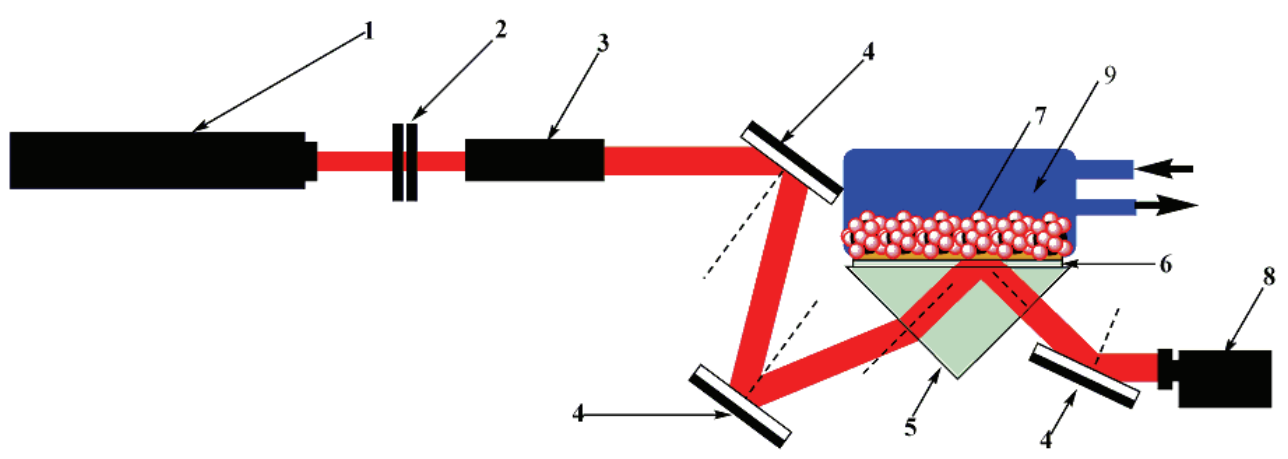

Fig. 4. SPRi modular set-up. Functioning principle - schematic view. 1 - He-Ne laser; 2 polarysors; 3 - collimator; 4 - tilting mirrors; 6 - drop of oil; 7 - PAMAM-containing sensor; 8 - CCD camera; 9 - flow cell with inlet and outlet for analyte circulation

\subsubsection{Mineralization occurrence}

The PAMAM-containing sensors are fixed on the SPRi analysis platform as displayed in Fig.4 and then exposed to the experimental fluids, inside the thermostated flow cell. PAMAM macromolecules create nanorough surfaces with affinity towards calcium ions (through internal amide, and external amino and/or carboxyl groups). These macromolecules present an exterior reactive shell bearing $-\mathrm{NH}_{2}$ or $-\mathrm{COOH}$ and an intrinsic nanoporosity due to their hyperbranched tree-like structures divergent from a central core (see Fig. 3). 
The architecture and the assembling of the molecules onto the gold surface are responsible for the creation of a porous layer expected to contain delineated cages in which mineral supersaturated solutions accumulates and determine the nucleation of the mineral phase.

The above mentioned functional groups (amide, amino and carboxyl) behave like ionic pumps capturing calcium ions from the test fluids; then calcium cations exert affinity towards phosphate anions and the repetition of that sequence generates calcium-phosphate mineral nuclei on the dendrimer surface; these inorganic structures develop themselves and continue to grow by ions capturing from the SBFs when the sensors are exposed to the mother liquors (left panels in Fig.5).

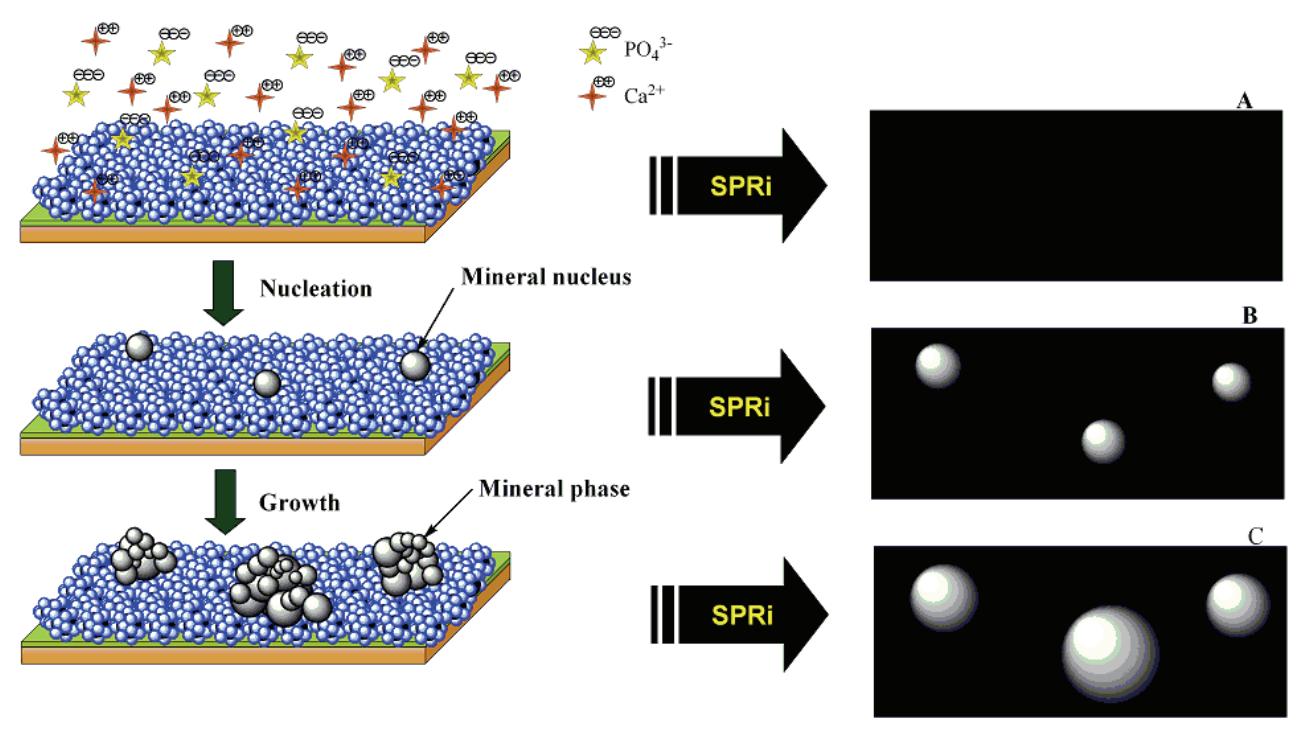

Fig. 5. Mineralization - occurrence and detection. Schematic view of: left - the evolution of the phenomenon with the formation of mineral clusters on the sensor's surface and right the associated SPRi images as detected at PAMAM-coated gold SPR angle: A-minimum reflectivity at PAMAM-coated gold SPR angle; B - bright area due to nucleation; C expanded bright spots due to mineral growth

\subsubsection{Mineralization detection principle}

The analysis tool SPRi is expected to be sensitive enough in order to provide important quantitative information on mineralization's occurrence and kinetics. The innovative SPRi detection of the mineralization is based on the specific mass change induced by the mineral nuclei formation and growth.

Of course, the technique is not adapted to distinguish between the origin/nature of the mass change, but only to sense the refraction index modification associated to that one. However, it should clearly be stated that, in that experiment, the only reason of mass change on the sensor's surface is the mineral precipitation due to nucleation and growth. These events are going to be detected and monitored as schematically presented in Fig.5.

The detection should be recorded at the SPR angle of the PAMAM-coated sensor (where the minimum reflectivity takes place leading to the darkest image of the whole sensor's surface) 
as shown in panel A from Fig.5. Following nucleation in well distinct areas of the sensor a corresponding modification of the refractive index occurs and leads to the signals recorded on the image as displayed in panel B fig.5. Increasing the amount of mineral phase during the nucleation and growth leads to more intense signals schematically presented in panel $\mathrm{C}$. The mineralization is not an instantaneous phenomenon. This is why, in a first attempt, the kinetics of the phenomenon should be explored in terms of induction time defined as the time, from the beginning of the experiment, needed to notice mineral nuclei formation (as the first detectable spots on the dark background). Since this might need long functioning time, it is recommended to operate the system at predetermined time intervals. Only then the experiment conditions may be optimized; data should be collected and then processed.

\section{Results and discussion}

\subsection{Biomineralization - general considerations}

Biomineralization represents the general phenomenon by which mineral formation occurs in living organisms. The process is extremely complex, it covers a multidisciplinary area and it presents specific features to each type of species involved; furthermore its nature (physiological or pathologic) and its localization in the body lead to distinct characteristics. Several researchers have tried a better understanding of the way this processes occurs. Without trying to explain here how and why biomineralization takes place, I would like to mention a reference book - On biomineralization written by Lowenstam and Weiner (Lowenstam \& Weiner, 1989) giving a wide and comprehensive background on the phenomenon.

In this chapter I would only like to refer to the importance of macromolecules in mineral formation inside organisms. More specifically, mineral formation in bones occurs on a previously formed organic matrix consisting mainly in collagen and containing noncollagenic proteins as well. The main actors of the biomineralization are represented by cellular and acellular components functioning in an aqueous environment, but "no specialized cellular or macromolecular machinery" is known as set-up to induce mineralization (Lowenstam \& Weiner, 1989); the phenomenon is generally induced even by minor perturbation of the liquid media. With respect to the acellular species involved in biomineralization, it is generally believed and demonstrated that naturally occurring polymers - mainly proteins from the hard tissue structure present special influences on the phenomenon. The functionality and the distribution of the reactive groups of these biopolymers play important roles in the induction of the nucleation and mineral growth. The use of PAMAM dendrimers offers the possibility to modify/modulate both the functionality and the distribution of the functional groups in the aim of enhancing the biomineralization response.

The here proposed experimental platform is based on homogeneous layers of PAMAM (different end-groups and different sizes/generations); more complex sensors may be designed, allowing the simultaneous investigation of several identical or different PAMAM. It is believed that PAMAM macromolecules will behave like ionic pumps with predefined shape and delineation cages, presenting affinity towards calcium and phosphate from the test fluid; the successive capturing of calcium and phosphate should lead the formation of hydroxyapatite(-like) mineral structures. The variation of parameters like the generation and the functionality should help the understanding of functional groups influence on biomineralization. It is expected that higher generation, corresponding of course to higher 
number of amide and amino or carboxylic ending groups, will lead to more intense calcification of the matrix. Differences between the effect of the different ending functionalities is also anticipated.

On the micro-patterned sensors containing both amino-PAMAM as well as carboxylicPAMAM, the real time imaging of the local reflectivity should give details about who of the two chemical groups is more efficient in inducing and sustaining mineralization. Nucleation induction time should be recorded as well as the mineral growth kinetics depending of the number and type of functional shell of the tested dendrimers. Nevertheless, the best responsive PAMAM coating will be further available for implant coating.

\subsection{Biomineralization - investigation tools - SPRi solution}

Mineralization induction and control represent still a top-challenge in the field of hard tissue repair and regeneration. The interest on bone mineral phase formation has its start early in the biomedical field; Bronn and Buetchli cited by Lowenstam and Weiner (Lowenstam \& Weiner, 1989) have performed an extraordinary work that marks the beginning of the biomineralization research, providing a solid background on mineralized structures despite their limited investigation tools: only light microscopy and chemical treatments. The modern biomineralization research started with the introduction of powerful tools such as X-ray diffraction and improved light microscopes combined with histological techniques that allow for improved access to tissue exploring. Other instruments and techniques have been added in time to the list of tools needed to help in morpho-functional biomineral and hard tissue evaluation: Scanning Electron Microscopy (SEM), Transmission Electron Microscopy (TEM), Micro-tomography. Energy dispersive X-ray spectrometer attached to electron microscopes (SEM or TEM) represents useful sensitive elemental analysis detector; however, it does not distinguish the mineral type. This latter task should be resolved with help from mineralogists. FT-IR spectroscopy and microscopy are nowadays also used to investigate the chemical structure of the biominerals.

In what concerns the induction of biomineral on different materials, sequential experiments have been presented in the literature. Basically the substrates believed to induce biomineral formation are immersed in one or several fluids with controlled composition, incubated under specific conditions (usually the physiological environment is mimicked) and at the end of the test the mineral formation is explored through the above mentioned techniques. Chemical analyses of the dissolved mineral are also an option, leading to information such as the molar ratios between different elements which in the case of calcium phosphates is very important (for instance a ration between $\mathrm{Ca}$ and $\mathrm{P}$ of 1.67 states for hydroxyapatite).

In order to obtain details on the biomineralization induction and kinetics, a high number of samples, important amounts of test materials and long experimental times are needed. To be more specific, an example will be presented. In a typical in vitro acellular biomineralization assay, in our group we use to explore at least 12 samples of each tested substrate; each sample is incubated in $50 \mathrm{ml} \mathrm{SBF}$, under physiological temperature and $\mathrm{pH}$, for at least 14 days (Stancu et al, 2004). Each 48 hours the incubation fluid is changed in order to provide enough ions available for the potential mineralization. At the end of the incubation time each sample is rinsed to remove non-bound mineral and debris and then the typical investigation of mineral formation is followed: SEM, TEM, EDS, FT-IR, chemical analyses, performed at least in triplicate (see details in Stancu et al, 2004).

Nevertheless, the analyses and the processing of the collected data at the end of the experiments are important. 
With this respect, the present work introduces a totally new approach, based on the evaluation of one sensor at the time, using an important amount of SBF incubation fluid with constant composition, since the fluid is continuously renewed onto the sensor surface. The fluid is continuously pumped on the sensor's surface using a peristaltic pump with controlled flow of $2 \mathrm{ml} /$ minute, like in natural bone tissue. Based on the investigator's expertise in what concerns the induction time (it is recommended to first explore the biomineralization induction time in order to start the detection just before its end), the sample can be monitored to record any mass change/refractive index change in real time. The samples are imaged and as soon as brighter spots appear on the dark background it can be concluded that the mineral nucleation took place. Then, increasing the intensity of the signals proves the mineral phase growth and spreading onto the tested material.

One major disadvantage of this method should be revealed here; it consists in the lack of specificity with regard to the chemical nature and complexity of the bound species. However, under controlled experimental conditions if the only source of mass change is represented by mineral formation, this disadvantage is minimized or even removed. Nevertheless, the sensor can be removed at the end of the experiment and its surface can be submitted to other specific investigation such as FT-IR or SEM to obtain complementary data on the deposited phase. A second problem associated to that method could be represented by too long operation times needed - this could be an important technical and economical problem when the light source consists in a He-Ne laser.

Now, that the most important disadvantages have been exposed, the advantages of the SPRi approach for the biomineralization investigation should be highlighted too. The high sensitivity of the SPRi tool, able to detect even few ppm of bound molecules, is an important parameter allowing to detect the presence of the early nucleation sites onto the surface of the sensor. This aspect is extremely useful since none of the previously presented techniques is able to identify that "at this moment is the real zero time and here is the zero point" of the mineral formation. Depending on the chemical nature and on the homogeneity of the analysed substrate one may explore the efficiency of a certain substrate against a different one, through micro-patterning of the sensor's surface followed by competitive monitoring of the places where the first nuclei appear (schematically displayed in Fig.6). The continuous flow of mother liquor needed to induce mineralization brings also a plus for the newly proposed method when compared with the classical ones. Typically, the old wide used methods based on the incubation of the samples in known amount of fluid (for instance $50 \mathrm{ml} \mathrm{-} \mathrm{(Stancu} \mathrm{et} \mathrm{al.,} \mathrm{2004))} \mathrm{and} \mathrm{the} \mathrm{change} \mathrm{of} \mathrm{that} \mathrm{one} \mathrm{at} \mathrm{regular} \mathrm{time} \mathrm{intervals} \mathrm{(e.g.}$ 48 hours) is not, of course, the best solution since the fluid may become poor in the needed ions and then the mineralization potential of the substrate may be misjudged. The new approach is using the continuous flow of fresh SBF and exposes the substrate to a considerable volume of $5768 \mathrm{ml}$ of testing fluid in only 48 hours, which, of course, represent an advantage with respect to the accuracy of the estimation. Moreover, thin layers and respectively low amounts of sensing phase are needed in this experiment. PAMAM dendrimers that are used in this study as sensing phase are quite expensive and the evaluation of their mineralization potential when immobilized on different scaffolds would need an important budget if performed using the classical methods. If choosing the SPRi here proposed experiment, the same or even better results may be obtained with considerably lower amount of dendrimers. Nevertheless, their assembling onto the sensor may be performed on micro-domains leading to both reproducibility and competitive 
results between different functionalities of the external shells in a simple experiment, on a 10 x 10 mm slide (see Fig.6).
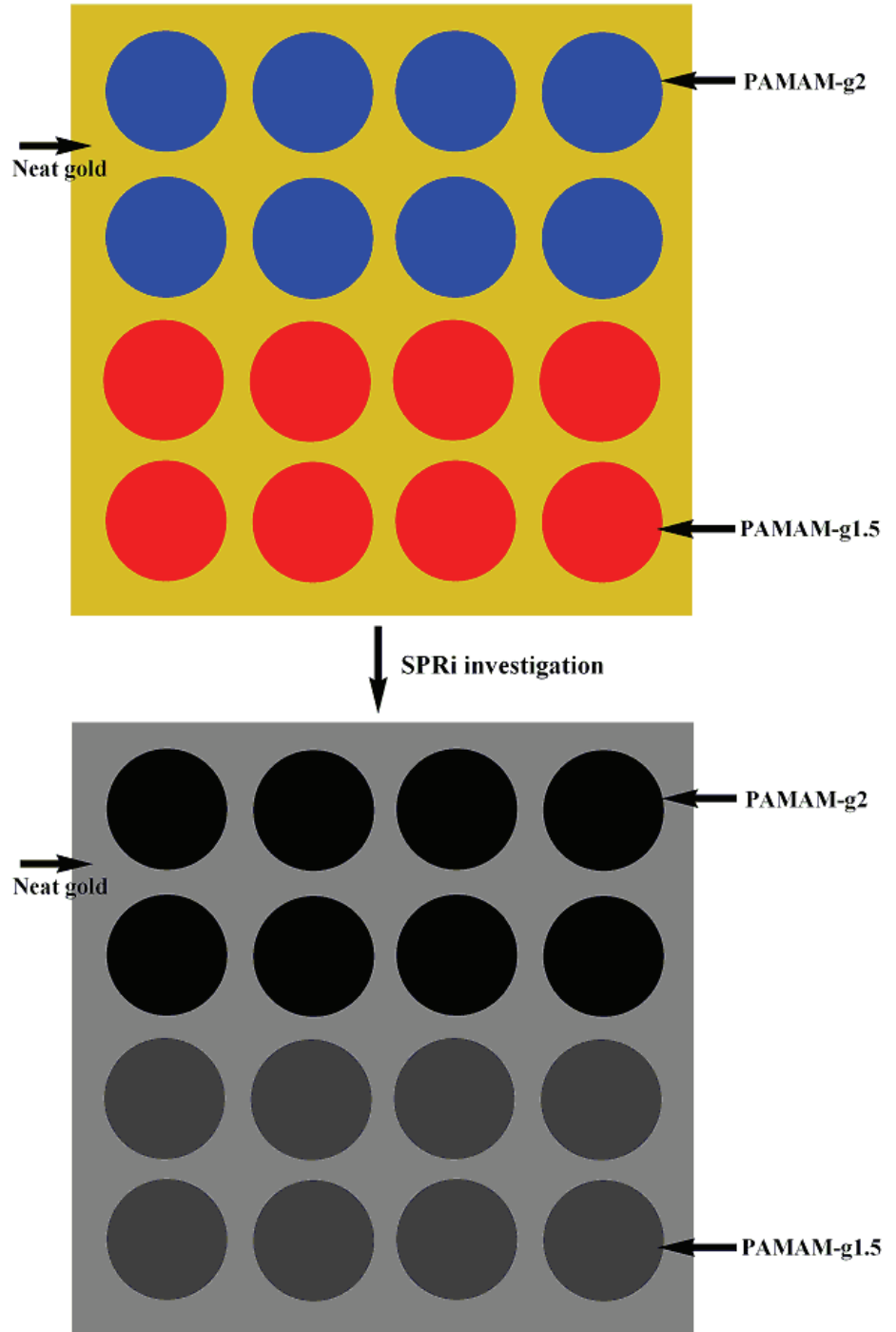

Fig. 6. Micro-patterned sensor bearing PAMAM-g2 and PAMAM-g1.5; up panel - sensor's schematic view; down - sensor imaged at SPR angle of the PAMAM-g2-coated substrate and under SBF before the nucleation occurs.

These practical aspects being mentioned, several design related features should be also emphasized. It is of maximum importance that the immobilization of the sensing phase takes place in a controlled manner, leading to ultrathin and homogeneous layers of dendrimers. Agglomeration of these macromolecules onto the sensor surface would lead to 
non-homogeneous signal that might make the observation of the mineralization impossible. Nevertheless, the important size of PAMAM molecules, increasing with the generation, limits the sensitivity of the sensor at too high generations due to surface's saturation. When micro-patterning is intended to check for reproducibility in the same experimental conditions, several spots of dendrimer should be created on the sensor; after exposure to the experimental fluid the results may be compared and the generated conclusion will be more precise. Moreover, the micro-paterning could allow for comparative studies between different immobilized species. In this latter case the start image will be similar to the schematic presented in Fig.6: darkest areas correspond to the PAMAM-g2-coated spots observed at their SPR angle under liquid; the images of the PAMAM-g1.5-coated spots appear brighter due to their different refractive index. When mineralization takes place, brighter signals will be noticed on the respective substrate.

The sensors may be designed/modified using different generation of dendrimers and different functionalities. This will enable comparative studies on the influence of the generation and of the external reactive shell on the mineralization occurrence. This information is extremely useful in further nano-patterning different implants with the best PAMAM coating.

\section{Conclusion}

The proposed SPRi assessment of the mineralization occurrence could be very useful in the better understanding of the biomineralization, providing information impossible to be revealed by the already existing techniques. The method is simple, clean, very sensitive and efficient, opening a new route to mineralization evaluation. Practical advantages are evident against some inherent disadvantages.

Versatility represents a major characteristic of this method since the set-up and the experimental conditions are easy to be changed depending on the investigator's choice. Nevertheless, the use of PAMAM nanostructured surfaces to induce biomineral formation has its novelty character too. Versatility is also the key attribute of the developed sensors since both the functionality and the generation of the dendrimers may be modified in order to get the best biomineralization answear.

To my knowledge, this is the first attempt of investigating mineralization through SPRi. Further development, improvements and experimental data will follow this approach.

\section{Acknowledgements}

The National Authority for Scientific Research from The Ministry of Education, Research and Youth of Romania is gratefully acknowledged for the financial support through the exploratory project "Polymeric Biomaterials for Bone Repair. Biomimetism through Nanostructured Surface", PN-II-ID-2008-2, number 729/19.01.2009

\section{References}

Donners J.J.J.M.; Nolte R.J.M.; Sommerdijk N.A.J.M. (2003). Control over calcium carbonate phase formation by dendrimer/surfactant templates. Advanced Materials, Vol.15, No. 4, 313-316, ISSN 0935-9648 (print) 1521-4095 (Online) 
Filmon R.; Grizon F.; Basle M.F.; Chappard D. (2002). Effects of negatively charged groups (carboxymethyl) on the calcification of poly(2-hydroxyethyl methacrylate). Biomaterials, Vol. 23, No.14 (July 2002), 3053-3059

Ganss B.; Kim R.H.; Sodek J. (1999). Bone sialoprotein. Critical Reviews in Oral Biology E Medecine, Vol 10 (January 1999) 79 -98, ISSN 1544-1113 (online) 1045-4411 (print),

Hunter G.K.; Poitras M.S.; Underhill T.M.; Grynpas M.D.; Goldberg H.A. (2001). Induction of collagen mineralization by a bone sialoprotein- decorin chimeric protein. Journal of Biomedical Materials Research, Vol. 55, No.4 (June 2001) 496-502, ISSN 1552-4965 (online) 1549-3296 (print)

Jaakkola, T.; Rich, J.; Tirri, T.; Narhi, T.; Jokinen, M.; Seppala, J.; Yli-Urpo, A. (2004). In vitro Ca-P precipitation on biodegradable thermoplastic composite of poly(Ecaprolactone-co-DL-lactide) and bioactive glass (S53P4). Biomaterials., Vol. 25, No. 4 (February 2004) 575-581, ISSN 0142-9612

Kamei S.M; Tomita N.; Tamai S.; Kato K.; Ikada Y. (1997) Histologic and mechanical evaluation for bone bonding of polymer surfaces grafted with a phosphatecontaining polymer. Journal of Biomedical Materials Research, Vol. 37, No.3 (December 1997) 384 -393, ISSN 1552-4965 (online) 1549-3296 (print)

Kokubo, T.; Ito, S.; Huang, Z. T.; Hayashi, T.; Sakka, S.; Kitsugi, T. and Yamamuro, T. (1990). Ca-P rich layer formed on high strength bioactive glass-ceramics. Journal of Biomedical Materials Research. Vol. 24, No.3 (March 1990) 331-343, ISSN 1552-4965 (online) 1549-3296 (print)

Lowenstam, H.A.; Weiner, S. (1989), On biomineralization, Oxford Academic Press, ISBN 019-504977-2 (U.S.), New York

Majoros, I.J.; Mehta, C.B.; Baker Jr., J. R. (2004). Mathematical Description of Dendrimer Structure. Journal of Computational and Theoretical Nanoscience, Vol. 1, No. 2 (September 2004), 193-198(6), ISSN 1546-1955, 1546-1963

Price, R.L.; Haberstroh, K.M. and Webster, T.J. (2003). Enhanced Functions of Osteoblasts on Nanostructured Surfaces of Carbon and Alumina. Medical and Biological Engineering and Computing (Incorporating Cellular Engineering), Vol. 41, No. 3 (May 2003) 372375, ISSN 0140-0118 (Print) 1741-0444 (Online)

Shin H.; Jo S.; Mikos A.G. (2003). Biomimetic materials for tissue engineering. Biomaterials Vol. 24, No.13 (...2003) 4353-4364, ISSN 0142-9612

Stancu, I. C.; Filmon, R.; Cincu, C.; Marculescu, B.; Zaharia, C.; Tourmen, Y.; Basle, M. F.; Chappard, D. (2004). Synthesis of methacryloyloxyethyl methacrylate phosphate copolymers and in vitro calcification capacity. Biomaterials, Vol. 25, No. 2 (January 2004) 205-213, ISSN 0142-9612

Stancu, I.C.; Fernandez-Gonzalez, A.; Salzer, R. (2007). SPR imaging antimucin-mucin bioaffinity biosensor as label-free tool for early cancer diagnosis. Design and detection principle. Journal of Optoelectronics and Advanced Materials, Vol. 9, No. 6 (June 2007) 1883-1889, ISSN 1454 - 4164, 1841 - 7132

Swart J.G.N.; Driessen A.A.; DeVisser A.C. (1976). Calcification and bone induction studies in heterogeneous pohosphorylated hydrogels. In: Hydrogels for medical and related applications. Andrade J.D. (Ed.), 151-161, ACS Symposium Series, No.31, Washington DC

Ulman, A. (1991), An Introduction to Ultrathin Organic Films from Langmuir-Blodgett to SelfAssembly, Academic Press, ISBN 0127082301, 9780127082301, 978-0127082301 
Vijayasekaran S.; Chirila T.V.; Robertson T.A.; Lou X.; Fitton J.H.; Hicks C.R.; Constable I.J. (2000) Calcification of poly(2-hydroxyethyl methacrylate) hydrogel sponges implanted in the rabbit cornea: A 3-month study. Journal of Biomaterials Science. Polymer Edition, Vol. 11, No.6 (June 2000) 599-615, ISSN 0920-5063, 1568-5624 (Online)

Ward B.C.; Webster T.J. (2006). The effect of nanotopography on calcium and phosphorus deposition on metallic materials in vitro, Biomaterials, 27 (June 2006) 575-8, ISSN 0142-9612

Whyte M.P.; Landt M.; Ryan L.M.; Mulivor R.A.; Henthorn P.S.; Fedde K.N.; Mahuren J.D.; Coburn S.P. (1995). Alkaline phosphatase: Placental and tissue-nonspecific isoenzymes hydrolyze phosphoethanolamine, inorganic pyrophosphate, and pyridoxal 5-phosphate. Substrate accumulation in carriers of hypophosphatasia corrects during pregnancy. Journal of Clinical Investigation, vol. 95 (April 1995) 14401445, ISSN 0021-9738 


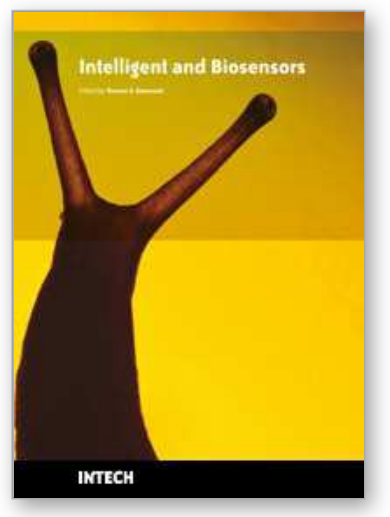

\author{
Intelligent and Biosensors \\ Edited by Vernon S. Somerset
}

ISBN 978-953-7619-58-9

Hard cover, 386 pages

Publisher InTech

Published online 01, January, 2010

Published in print edition January, 2010

The use of intelligent sensors have revolutionized the way in which we gather data from the world around us, how we extract useful information from that data, and the manner in which we use the newly obtained information for various operations and decision making. This book is an attempt to highlight the current research in the field of Intelligent and Biosensors, thereby describing state-of-the-art techniques in the field and emerging new technologies, also showcasing some examples and applications.

\title{
How to reference
}

In order to correctly reference this scholarly work, feel free to copy and paste the following:

Stancu Izabela-Cristina (2010). SPR Imaging Label-Free Control of Biomineral Nucleation!?, Intelligent and Biosensors, Vernon S. Somerset (Ed.), ISBN: 978-953-7619-58-9, InTech, Available from: http://www.intechopen.com/books/intelligent-and-biosensors/spr-imaging-label-free-control-of-biomineralnucleation

\section{INTECH}

open science | open minds

\section{InTech Europe}

University Campus STeP Ri

Slavka Krautzeka 83/A

51000 Rijeka, Croatia

Phone: +385 (51) 770447

Fax: +385 (51) 686166

www.intechopen.com

\section{InTech China}

Unit 405, Office Block, Hotel Equatorial Shanghai

No.65, Yan An Road (West), Shanghai, 200040, China

中国上海市延安西路65号上海国际贵都大饭店办公楼 405 单元

Phone: +86-21-62489820

Fax: $+86-21-62489821$ 
(C) 2010 The Author(s). Licensee IntechOpen. This chapter is distributed under the terms of the Creative Commons Attribution-NonCommercialShareAlike-3.0 License, which permits use, distribution and reproduction for non-commercial purposes, provided the original is properly cited and derivative works building on this content are distributed under the same license. 